\title{
RENDICIÓN DE CUENTAS EN LAS ONG: ¿A QUIÉN Y POR QUÉ DEBEN RENDIR CUENTAS?
}

\author{
NGO ACCOUNTABILITY: TO WHOM AND WHY SHOULD THEY BE \\ ACCOUNTABLE?
}

Martha Adriana Chávez Passano Universidad del Pacífico Lima, Perú

ORCID: http://orcid.org/0000-0003-2654-6207

Correo electrónico: chavez_ma@up.edu.pe

\section{RESUMEN}

El aumento significativo del número de Organizaciones no Gubernamentales (ONG) activas, la cantidad de bienes que manejan y los diferentes grupos interesados en sus actividades las obliga a ser transparentes. La literatura que se ha revisado nos ayuda a entender, en primera instancia, a quiénes deben rendir cuentas las ONG; al respecto, encontramos diferentes grupos de stakeholders (gobierno, donantes, beneficiarios, entre otros). Actualmente, las organizaciones deben buscar incorporar el accountability como parte de su estrategia. No solo se debe rendir cuentas en el ámbito financiero, sino también informar sobre sus actividades, su gestión, su estrategia y su dirección. El accountability, además, debe tener un enfoque interno, no solamente hacia fuera, con el fin de buscar la participación de los interesados. Finalmente, será posible identificar las principales formas de categorización del accountability.

Palabras clave: ONG; rendición de cuentas; grupos de interés; estrategia.

\begin{abstract}
The significant increase in the number of active Non-Governmental Organizations (NGOs), the amount of goods they manage and the different groups interested in their activities, oblige them to be transparent. The literature that will be reviewed helps us to understand, in first place, to whom should NGOs be accountable to, where we find different groups of stakeholders (government, donors, beneficiaries, among others). Nowadays organizations should seek to incorporate accountability as part of their strategy. Accountability should not be only conducted in the financial sphere, but they must also report on their activities, their management, their strategy and their direction. Accountability must also have an internal focus, seeking the participation of interested parties. Finally, we can identify the main forms of accountability categorization.
\end{abstract}

Keywords: NGOs; accountability; stakeholders; strategy.

(c) Los autores. Este artículo es publicado por la Revista Quipukamayoc, Universidad Nacional Mayor de San Marcos. Este es un artículo de acceso abierto, distribuido bajo los términos de la Licencia Creative Commons Atribución-NoComercial-Compartirlgual 4.0 Internacional.(http://creativecommons.org/licenses/by-nc-sa/4.0/), que permite el uso no comercial, distribución y reproducción en cualquier medio, siempre que la obra original sea debidamente citadas. 


\section{INTRODUCCIÓN}

El propósito de esta investigación es analizar e identificar en qué medida las Organizaciones No Gubernamentales (ONG) saben cómo, por qué y a quién deben rendir cuentas. Accountability en las ONG es la respuesta a la inconsistencia entre los objetivos y los valores de las organizaciones sin fines de lucro y las personas e instituciones que tienen relación directa o indirecta con ellas, las cuales proporcionan información incompleta y de poca credibilidad o peor aún, no ofrecen ningún dato sobre sus resultados, no solo financieros, sino también sobre el impacto en la sociedad y en el grupo beneficiario.

Para que estas organizaciones sean sostenibles en el tiempo, no solamente en el ámbito financiero, es importante que la sociedad, como un todo, legitime a las ONG y confíe en ellas, lo que implica reconocerlas como organizaciones con valores que las acercan a sus objetivos y con transparencia en sus acciones.

En los últimos años se ha incrementado notablemente el número de ONG con un amplio espectro de programas y objetivos, con diversos niveles de financiamiento por parte de organizaciones internacionales y el sector privado nacional. Por tanto, se han canalizado, a través de las ONG, montos significativos de ayuda, en forma de dinero o bienes, lo que las obliga a ser más responsables en cuanto a su transparencia, no solo respecto de sus donantes sino también de los demás grupos de interés o stakeholders, como lo son los donantes independientes, los beneficiarios, el gobierno, etcétera.

Esta preocupación respecto a la rendición de cuentas y a la transparencia de las ONG nos lleva a replantear el rol de estas organizaciones, que hoy en día son consideradas entidades que contribuyen con el desarrollo y que aportan a la democratización. Entonces, deben, como cualquier organización pública o privada, con o sin fines de lucro, tener un buen desempeño y ser eficientes para poder lograr su sostenibilidad y legitimidad dentro de la sociedad.

Los recientes sucesos de corrupción y poca transparencia de la información y de la gestión de dichas organizaciones confirman la necesidad de luchar para que las ONG en el Perú rindan cuentas. Dada esta coyuntura, es posible notar que las propias organizaciones están reaccionando, pero necesitan apoyo para poder estudiar e implementar las buenas prácticas de accountability.

Actualmente, los sectores público, privado y civil exigen que se examine la rendición de cuentas en las ONG. Ello genera algunas dudas - aún no resueltas - acerca de los métodos de rendición de cuentas, los derechos de quienes la solicitan y las formas de presentarla.
Enseguida se revisará la literatura más relevante sobre el concepto de rendición de cuentas o accountability y su importancia para las ONG, lo que nos permitirá comprender estos conceptos, así como ayudarnos en el estudio propuesto. Luego, se planteará la pregunta, ¿a quién y por qué las ONG deben rendir cuentas?

\section{Revisión de literatura}

Sobre la rendición de cuentas (accountability). Accountability es una palabra muy utilizada en las organizaciones no lucrativas del mundo anglosajón, una de sus características es que trata de expresar la necesidad que tienen estas organizaciones de dar respuesta a sus actuaciones. Entonces, podemos entender la palabra accountability como rendición de cuentas o explicación de la actuación de la organización.

Por un lado, es posible entender la rendición de cuentas como la actitud de asumir la responsabilidad por las acciones que realizamos y, por otro, como la posibilidad de asegurar, en caso se tenga un desempeño deficiente o fuera de lo correcto, la aplicación de sanciones o castigos para corregir lo actuado (Goetz y Jenkins, 2001).

Asimismo, no se debe caer en la idea de pensar que dar explicaciones se refiere solo a la rendición de las cuentas económicas y contables de la organización. Ello debe entenderse como la responsabilidad que tiene la organización de explicar y de informar a todos los que se relacionan con ella $-\mathrm{y}$ en general, a la sociedad- de todas las acciones que se han realizado y, además, mostrar sus resultados y si se han alcanzado las metas establecidas durante un periodo en particular. En suma, todo ello implica ser transparentes y creíbles ante la sociedad en su conjunto (Vernis, 2004).

Así pues, se entiende por rendición de cuentas a la responsabilidad que asumen las organizaciones respecto a las acciones que realizan, pero esto, además, genera algunas interrogantes que no siempre son fáciles de responder, como, por ejemplo, ¿cuál es el propósito de la rendición de cuentas? ¿Quiénes deben presentar la rendición de cuentas? ¿Sobre qué rendir cuentas y ante quiénes hacerlo? (Cornwall, Lucas \& Pasteur, 2000).

Podemos observar dos definiciones de accountability, en primer lugar, una definición concreta que se sustenta únicamente en responder a lo legal y a lo obligatorio; la otra es una definición amplia que busca dar respuesta a la sociedad en general, por lo cual amplía sus explicaciones a todo lo que afecta a la organización (Kearns, 1996).

El propio Kearns (1996) define la palabra accountability como sigue: "Significa conservar la confianza del público al mismo tiempo que se es capaz de explicar las promesas hechas por la organización a las personas que la apoyan, 
todo ello sin alejarse de la misión y con prácticas de gestión correctas" (p. 90).

El concepto de rendición de cuentas se ha definido de distintas formas, y no todas ellas son compatibles o complementarias. Una perspectiva desde la que esta puede observarse, implica verla como la responsabilidad de responder por un desempeño particular ante las expectativas de distintas partes interesadas. Por ello, la rendición de cuentas se puede concentrar en demandas específicas; por ejemplo, sobre informes financieros, sobre los servicios que la organización brinda, sobre las tácticas utilizadas en una campaña, entre otras. Así pues, los valores, la misión y las estrategias de las organizaciones de la sociedad civil definen los objetivos y las actividades sobre los cuales deben rendir cuentas a las distintas partes interesadas (Brown, 2005).

Es necesario que se definan indicadores de conducta, de desempeño y otros parámetros de evaluación para lograr el accountability. De esta manera, se podrán manejar las relaciones sociales y las expectativas de los stakeholders o grupos de interés. Por consiguiente, la construcción de una rendición de cuentas eficiente involucra - directamente - tener bien definidas las relaciones entre los distintos actores y el conocimiento de sus posiciones de poder. Todos los involucrados deben tener claro quién está en el derecho de exigir cuentas y quién está en el deber de explicar sus actos (Day y Klein, 1987).

\section{Accountability en las ONG}

En relación con la rendición de cuentas o accountability en la ONG, Vernis (2004) sostiene que "esta es la necesidad que tienen estas organizaciones de explicar con rigurosidad sus acciones" (p. 176). Lo que sustenta esta necesidad es muy sencillo y directo, las ONG deben generar confianza respecto a sus acciones ante la sociedad, en la que ocupan un espacio importante $y$, en muchos casos, cumplen un rol protagónico que las hace responsables frente a todos y ante su propia organización.

Por lo anterior, entendemos la importancia de la rendición de cuentas de las ONG, pero ¿cómo proceder?, ¿cómo aplicarla? Para ello, se debe identificar, en primer lugar, a quiénes debemos rendir cuentas y de qué.

Vernis (2004) señala que:

No se puede generalizar, ya que cada organización deberá identificar a quiénes rendir cuentas y de qué $y$ cuál debe ser el alcance en cada caso $y$, de ser necesario, priorizar a quién y qué información presentar. Este autor propone cinco grandes áreas sobres las que las ONG tienen que rendir cuentas: área financiera, área de gestión, área de actividades, área directiva y área estratégica. (p. 182)

En la tabla 1 se presenta el contenido de cada una de ellas.

Vernis (2004) define estas cinco áreas, a las que considera básicas. Asimismo, podemos observar que no solo se limita a dar información sobre temas financieros o contables, aunque para muchos ello sería suficiente. En suma, no solo se trata de definir sobre qué rendir cuentas sino definir cómo y cuándo hacerlo y con qué periodicidad informar.

No hay una única respuesta en cuanto a quiénes debemos rendir cuentas como ONG. No obstante, puede utilizarse la siguiente generalización para identificar a las personas y grupos, a los que podemos dividir con un criterio básico que nos ayudará a ordenarlos: grupos internos y grupos externos (AbouAssi y Trent, 2016).

Al no tener suficientes recursos, una ONG logra subsistir y desarrollar su estrategia mediante la adquisición y mantenimiento de aquello que posee. Estos recursos son controlados por actores externos, quienes tienen derecho

Tabla 1

Áreas de Rendición de Cuentas.

\begin{tabular}{|c|c|}
\hline Área & Contenido \\
\hline Área financiera/fiscal & $\begin{array}{l}\text { Se tratará de explicar detalladamente el buen uso que realiza ONG de todas las aportaciones que } \\
\text { recibe. }\end{array}$ \\
\hline Área de gestión & Su objetivo es demostrar la utilización de procedimientos de gestión adecuados para su trabajo. \\
\hline Área de actividades & $\begin{array}{l}\text { La organización deberá explicar cómo realiza sus actividades y proyectos para demostrar que trata de } \\
\text { implementarlos siempre con la máxima calidad posible. }\end{array}$ \\
\hline Área directiva & $\begin{array}{l}\text { Se debe explicar qué personas forman parte de los órganos de gobierno, quienes dirigen la organiza- } \\
\text { ción y quiénes trabajan en ella. }\end{array}$ \\
\hline Área estratégica & $\begin{array}{l}\text { La organización tratará de demostrar que enfoca su trabajo hacia los aspectos más relevantes que } \\
\text { dicta la misión. }\end{array}$ \\
\hline
\end{tabular}

Fuente: Vernis (2004, p. 182). 
a solicitar la información que requieran. A través de mecanismos de accountability, se pueden amortiguar las demandas de actores externos y, de esta manera, gestionar sus expectativas.

En otros casos, ocurre que los actores externos buscan beneficiarse de la experticia de los agentes de la organización para conducir sus intereses (Moe, 1984). El gran problema de este mecanismo surge cuando los actores no saben lo que los agentes están haciendo o confían en ello. En esta coyuntura puede usarse la definición de accountability como "el derecho de los actores a exigir una rendición de cuentas de los agentes y también el derecho a imponer sanciones si las cuentas o acciones contabilizadas son inadecuadas" (Ebrahim, 2003, p. 813).

El accountability externo se asocia a múltiples dimensiones. Así pues, el upward accountability es la relación entre las ONG, los donantes y el gobierno respecto al control sobre los recursos financieros y regulatorios. Por su parte, el downward accountability es la relación entre las ONG y sus beneficiaros o cualquier persona o entidad que sea afectada indirectamente por sus acciones. Finalmente, el horizontal accountability es la relación que se tiene con organizaciones similares y que se demuestra usualmente a través de los mecanismos de autorregulación. En general, se puede decir que existe un sistema interrelacionado de responsabilidades y no un conjunto de relaciones que no se conectan una con la otra (Ebrahim, 2003).

AbouAssi (2013) sugiere que las ONG pueden reaccionar de cuatro maneras distintas a las prioridades de los donantes, quienes las presionan para cumplir con sus propias agendas e intereses. Salida es cuando una ONG decide no buscar más fondos de un donante en particular y suspender las relaciones. Voz se da cuando una ONG expresa sus comentarios y preocupaciones ante un donante, con la intención de influir en sus decisiones, a la vez que mantiene la relación y negocia un equilibrio entre los intereses de ambos actores. Lealtad es el cumplimiento instantáneo de una ONG con el donante, sin embargo, frecuentemente en las ONG que están organizadas por los donantes, en las que existe una falta de vinculación entre la organización y los contribuyentes. Finalmente, el ajuste es cuando la organización decide, voluntaria y deliberadamente, adaptar sus actividades y estrategia a favor de los nuevos objetivos del donante con el fin de asegurar el financiamiento.

La Figura 1 nos muestra, cuanto más fuerte es la responsabilidad interna hacia los valores y la misión, es más probable que una ONG pueda defenderse ante cualquier cambio relacionado al financiamiento de los donantes, lo que se ve reflejado en la salida. Además, cuanto más fuerte es la rendición de cuentas hacia abajo o downward accountability, es más fácil para la ONG utilizar las conexiones con las comunidades locales, lo que la diferencia de otras organizaciones empoderándola para ejercer su voz con los donantes. Mientras más financiamiento obtenga una ONG sin alterar actividades, es más probable que la organización defienda los intereses de las partes interesadas de la comunidad y, en consecuencia, refuerce el downward accountability. Cuando se da el caso de que una ONG cumple los requerimientos de los donantes sin expresar comentarios o preocupaciones, estamos hablando de una reacción de lealtad que se relaciona con el upward accountability. En muchos casos esto puede llegar a un equilibrio donde la organización se adapta a los requerimientos de los donantes y financiadores, pero al mismo tiempo manteniendo una relación cercana a las comunidades y otros interesados.

El accountability en una ONG también puede ser utilizado como estrategia de recursos. Muchas ONG carecen de recursos financieros adecuados para poder operar y suelen valerse de sus donantes para funcionar correctamente, servir a sus beneficiarios y ser sostenibles en el tiempo. Usualmente, las ONG enfocan su atención en la rendición de cuentas funcional para el uso de recursos y resultados

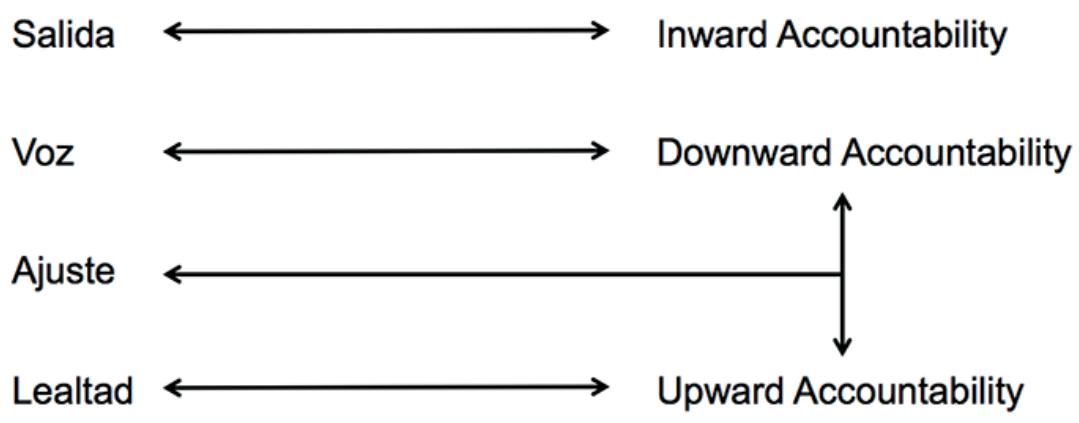

Figura 1. Interacción entre estrategias y responsabilidades de las ONG.

Fuente: Elaboración propia. 
inmediatos y, en menor medida, lo hacen de manera estratégica para generar impacto a largo plazo y sostenibilidad.

Los actores externos buscan promover un mayor profesionalismo, transparencia y eficiencia en las ONG; en ocasiones, en detrimento de la flexibilidad, la sensibilidad cultural y la rápida respuesta (Martens, 2008).

La dimensión interna del accountability en las ONG es ignorada con frecuencia. En este caso, la responsabilidad no es exigida desde afuera, sino que se genera dentro de la organización, esta es "motivada por la 'responsabilidad sentida' y expresada a través de las acciones individuales y la misión organizacional" (Ebrahim, 2003, p. 814).

Los procesos internos incluyen la participación de los ciudadanos, los beneficiarios y los donantes, y generan responsabilidad y mejoran el aprendizaje (Dubnick, 2011). Asimismo, más allá de ser únicamente responsables ante los stakeholders y demás interesados, se crean diálogos que reflejan la necesidad de desarrollo creativo y compromisos con los demás en una comunidad moral (Harmon, 1995).

Un siguiente paso será identificar específicamente en cada ONG quiénes son estos stakeholders y sobre qué rendir cuentas.

Kearns (1996) presenta otra forma de clasificar la rendición de cuentas. Para ello define dimensiones sobre las cuales las ONG pueden ordenar la información que van a explicar o mostrar, estas dimensiones son cuatro y las presenta como "respuestas": respuestas legales, respuestas negociadas, respuestas discrecionales y respuestas anticipadas.

Para poder dar las respuestas que Kearns (1996) plantea, también propone algunas preguntas que, en principio, pueden usar las ONG como guías en cada una de estas dimensiones.

En primer lugar, para las repuestas legales, las preguntas que pueden ayudar a encontrarlas pueden ser, por ejemplo: ¿qué regulaciones tiene que cumplir la organización? o ¿quién obliga a cumplir estas regulaciones? No basta con responder ambas interrogantes; lo importante es que las organizaciones puedan ser capaces de dar información más allá de lo obligatorio; es decir, que vayan más allá de las repuestas legales obligatorias, lo cual les permitirá generar confianza sobre sus acciones.

En cuanto a la segunda dimensión referente a las respuestas negociadas, estas se vinculan con las regulaciones o estándares que no necesariamente están definidos en las normas o leyes actuales.

Respecto a las respuestas discrecionales, estas, a diferencia de las dos primeras, no se relacionan con lo legal ni con lo obligatorio, sino con el criterio o juicio profesional de quienes dirigen o son responsables de las ONG, quienes pueden identificar qué respuestas son convenientes o necesarias para presentar a quienes corresponda, con el fin de generar confianza y legitimidad en la sociedad.

Por último, la dimensión de las respuestas anticipadas es aquella que considera situaciones que se han identificado en las ONG, que no necesariamente están planteadas en el momento, sino que les permiten adelantarse a futuras coyunturas que luego se convertirán en lo regular o establecido.

Estas dimensiones permiten que las ONG con pocos años de creación avancen con el modelo en el tiempo, en un inicio, estas se concentrarán en dar respuestas legales y luego ampliarán sus respuestas a las otras dimensiones. De esta manera el modelo permite que las ONG avancen a su ritmo de crecimiento y puedan ser organizaciones capaces de autoevaluarse, preguntarse por la importancia de cada dimensión y, en consecuencia, trabajar de manera particular cada una de ellas, de acuerdo con sus realidades y según con quiénes interactúan (Kearns, 1996).

La responsabilidad que tienen las ONG respecto a sus stakeholders va en dos direcciones: hacia abajo cuando involucra a los grupos beneficiarios, los empleados y los simpatizantes; y hacia arriba cuando implica al directorio, al gobierno y a los donantes. En la medida que una ONG pueda establecer prioridades claras y llegar a una concertación en sus numerosas responsabilidades, las rendiciones de cuentas hacia los grupos de interés dejarán de ser una preocupación. Se tiene la percepción general de que las ONG, al carecer de un fin de lucro, tienen menos intereses que los sectores privado y público, y que trabajan con una visión a largo plazo. De esta manera, se les permite, de manera tácita, ser representantes de los que no tienen voz al momento de tomar decisiones, como lo son los pobres, las nuevas generaciones y el medio ambiente (Edwards y Hulme, 1996).

\section{Categorización del accountability}

Se pueden distinguir dos tipos de rendición de cuentas: funcional y estratégica. La funcional tiene que ver con rendir cuentas sobre lo que ocurre a corto plazo, el empleo de recursos y su impacto inmediato. Por su parte, la estratégica implica, sobre todo, lo que ocurre a mediano y largo plazo; por ejemplo, la manera en que una ONG influye en los objetivos de otras organizaciones (Edwards y Hulme, 1996).

Otra clasificación separa la rendición de cuentas en tres categorías distintas: ante los patrones, ante los clientes y ante ellas mismas. Históricamente, los principales solicitantes de información sobre la actuación de las ONG han sido los donantes y, si bien esta predominancia se man- 
tiene, las otras dos categorías son consideradas muy importantes en la mayoría de estudios. Se suele confundir la rendición de cuentas con conceptos básicos y muy delimitados de evaluación y monitoreo de proyectos, algunos incluso opinan que discutir la rendición de cuentas se centra directamente en requerimientos que han sido establecidos por los donantes (Najam, 1996).

Existen niveles para organizar la rendición de cuentas de las ONG dependiendo ante quienes nos dirigimos, al personal operativo, a los miembros activos, a las contrapartes aliadas y a la comunidad, todo ello en función de las obligaciones presentes en la misión de la organización. Por otro lado, existen factores externos que inciden en la forma de rendir cuentas, factores como el cambio constante de las metas institucionales, el incremento de ingresos y el crecimiento desordenado de la organización los cuales pueden debilitar los mecanismos de redición de cuentas interno y afectar el desempeño de la misma (Edwards y Hulme, 1996).

Edwards y Hulme (1996) consideran necesario cumplir con ciertas condiciones para que las ONG puedan implementar una efectiva rendición de cuentas. Entre ellas destacan: el planteamiento de objetivos claros que orienten las acciones de la organización, los mecanismos de transparencia referentes a la toma de decisiones, la presentación de informes claros y fidedignos del uso de los recursos y las metas alcanzadas y, por último, los procesos de revisión y control y mecanismos de rendición de cuentas que permitan aplicar sanciones y recompensas cuando corresponda.

En la misma línea, Fowler (2000) considera que es importante y relevante contar con medidas e indicadores para corroborar los logros o metas alcanzadas, pues ello permitirá relacionar la rendición de cuentas con los valores y las normas de las ONG.

Por lo general, las evaluaciones dan importancia y se concentran solo en los resultados visibles a corto plazo y en el cumplimiento legal y fiscal. Por ejemplo, los donantes, en principio, dan preferencia a los reportes contables más que a una rendición de cuentas integral (Edwards y Hulme, 1996).

\section{CONCLUSIONES}

Luego de revisar la literatura más relevante, es posible identificar ciertas situaciones respecto a quiénes y por qué una ONG debe rendir cuentas.

Es fundamental que una ONG identifique claramente a sus stakeholders y que comprenda la relevancia de brindarles la información que requieran. Si una ONG logra identificar a sus stakeholders, pero no brinda información, ya sea por desconocimiento o malas prácticas, perderá confianza y su percepción se verá afectada. Esto podría ocasionar que se retiren sus fuentes de financiamiento, lo que puede comprometer a la organización y a sus actividades. En suma, las buenas relaciones y la comunicación con los stakeholders crearán valor para la organización.

Cabe acotar que existen distintas estrategias mediante las cuales las ONG pueden organizarse para saber a quiénes rendir cuentas. Una vez que estos actores han sido identificados, pueden utilizarse estas guías para determinar qué información debe ser compartida. Así pues, no solo es preciso informar acerca de las finanzas de la organización, sino también de las actividades, de la estrategia, etcétera.

Finalmente, tanto el accountability externo e interno son necesarios e importantes. Hacia afuera, se debe proveer la información que los stakeholders requieran; y dentro de la organización, se debe rendir cuentas a los actores internos para mejorar directamente el desempeño de la ONG.

\section{REFERENCIAS BIBLIOGRÁFICAS}

AbouAssi, K. (2013). Hands in the pockets of mercurial donors: NGOs response to shifting funding priorities. Nonprofit and Voluntary Sector Quarterly, 42(3), 584602. doi: https://doi.org/10.1177/0899764012439629

AbouAssi, K., \& Trent, D. (2016). NGO Accountability from an NGO Perspective: Perceptions, Strategies, and Practices. Public Administration and Development, 36(4), 283-296. doi: https://doi.org/10.1002/pad.1764

Brown, L. (2005). La construcción de la legitimidad y la rendición de cuentas en la sociedad civil. En C. Sanborn y F. Portocarrero (Eds.), Filantropía y cambio social en América Latina (pp. 441-460). Lima: Universidad del Pacífico.

Cornwall, A., Lucas, H., \& Pasteur, K. (2000). Introduction: Accountability through Participation: Developing Workable Partnership Models in the Health Sector. IDS Bulletin, 31(1), 1-13. doi: https://doi.org/10.1111/j.1759-5436.2000.mp31001001.x

Day, P., \& Klein, R. (1987). Accountabilities: Five Public Services. Serie Social Science Paperbacks. Londres: Tavistock Publications.

Dubnick, M. (2011). Move over Daniel, We Need some "Accountability Space". Administration and Society, 43(6), 704-716. doi: https://doi org/10.1177/0095399711424569

Ebrahim, A. (2003). Accountability in Practice: Mechanisms for NGOs. World Development, 31(5), 813-829. doi: 10.1016/S0305-750X(03)00014-7 
Edwards, M., \& Hulme, D. (1996). Beyond the Magic Bullet: NGO Performance and Accountability in the PostCold War World. Connecticut: Kumarian Press.

Fowler, A. (2000). Civil Society, NGDOs and Social Development: Changing the Rules of the Game. UNRISD Occasional Paper 1. Ginebra: United Nations Research Institute for Social Development.

Goetz, A., \& Jenkins, R. (2001). Hybrid Forms of Accountability: Citizen Engagement in Institutions of Public Sector Oversight in India. Public Management Review, 3(3), 363-383. doi: https:/doi. org/10.1080/14616670110051957

Harmon, M. (1995). Responsibility as Paradox: A Critique of Rational Discourse on Government. California: Sage Publications.
Kearns, K. (1996). Managing for Accountability. San Francisco: Wiley y Jossey-Bass.

Martens, K. (2008). Civil Society, Accountability and the UN System. Documento de trabajo CSGR 237/08. Coventry: Centre for the Study of Globalisation and Regionalisation.

Moe, T. (1984). The New Economics of Organizations. American Journal of Political Science, 28(4), 739-777. doi: $10.2307 / 2110997$

Najam, A. (1996). NGO Accountability: A Conceptual Framework. Development Policy Review, 4, 339-353. doi: https://doi.org/10.1111/j.1467-7679.1996.tb00112.x

Vernis, A. (2004). Los retos en la gestión de las organizaciones no lucrativas. claves para el fortalecimiento institucional del tercer sector. Barcelona: Granica. 
